\title{
Review and Proposed Management of Violence in the Secure Psychiatric Ward
}

ISSN: 2637-7934

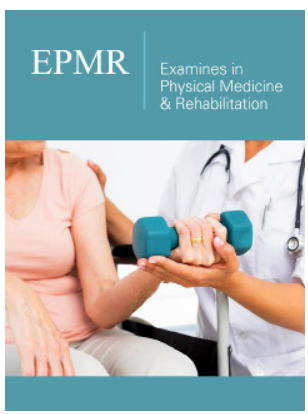

*Corresponding author: Ahmed Saeed Yahya, Specialist Trainee in Psychiatry, UK

Submission: 阱 May 21, 2019

Published: 海June 17, 2019

Volume 2 - Issue 4

How to cite this article: Ahmed Saeed Yahya , Simon Williams, Jude Ezeonwuka, Jude Chukwuma. Review and Proposed Management of Violence in the Secure Psychiatric Ward. Examines Phy Med Rehab.2(4). EPMR.000543.2019.

DOI: 10.31031/EPMR.2019.02.000543.

Copyright@ Ahmed Saeed Yahya, This article is distributed under the terms of the Creative Commons Attribution 4.0 International License, which permits unrestricted use and redistribution provided that the original author and source are credited.

\author{
Ahmed Saeed Yahya ${ }^{1 *}$, Simon Williams ${ }^{2}$,Jude Ezeonwuka ${ }^{3}$ and \\ Jude Chukwuma ${ }^{3}$ \\ ${ }^{1}$ Specialist Trainee in Psychiatry, UK \\ ${ }^{2}$ Consultant Forensic Psychiatrist, UK \\ ${ }^{3}$ Consultant Psychiatrist, UK
}

\begin{abstract}
Anger, hostility, aggression and violence are commonly encountered in the secure psychiatric wards. These acts interfere with the therapeutic milieu and cause unrest amongst both staff and patients. Understanding the aetiology and variables associated with these are critical to establishing safer wards. In this review article we amalgamate and summarize findings from research within the last ten years. We use evidence from recent research, clinical guidelines and our individual experiences to describe how simple interventions may help in reducing the incidence in locked wards.
\end{abstract}

Keywords: Anger; Hostility; Aggression; Violence; Secure wards

\section{Introduction}

Anger, hostility, aggression and violence are all interlinked, whilst commonly encountered within inpatient psychiatric environments. Escalation and difficulty in containing the above, normally leads to referral to services which offer greater levels of security (i.e. relational, environmental and procedural security). The Epidemiology Catchment Area study found that incidents of violence were five times higher in subjects with psychiatric illness [1]. There is some evidence from birth cohorts to suggest that there is a greater risk of violence amongst subjects with serious mental illness, in particular those with a diagnosis of Schizophrenia, when compared to the general population; this, however, remains a much debated, controversial finding [2]. It is therefore imperative to recognize and acknowledge these acts and the potential repercussions.

The goal of the inpatient setting is to provide a safe environment to aid the pathway to recovery. The therapeutic milieu is, however, very much unsettled by disturbing behaviors which place both the aggressor and other patients/staff at risk. The effect of these behaviors is extensive; from previous work, we are aware of the psychological impact of this on nursing staff [3] Dealing with these troubled behaviors on a daily basis can contribute to burnout in staff [3]. Our aim is to review the current research in the aetiology and variables associated with anger and hostility, which in turn drives aggression in these settings; our focus remains on the secure (PICU and Forensic) wards. There appear similarities in the antecedents which lead to violence by patients who are admitted to the wards, regardless of their psychiatric diagnosis. We will discuss briefly how this can be prevented with particular emphasis on the beneficial effects of changing the ward structure, organization and design.

To aid our review a literature search was conducted on articles produced in the last ten years around the aetiology of anger/hostility and violence. Our search yielded three sets of guidelines from NICE which included short term management, and the prevalence of violence/aggressive behaviors in those with a mental health diagnosis. We found two systematic reviews which focused on aggression in psychiatric wards and three research articles investigating the aetiology. 
Aetiology and Variables Associated with Anger, Hostility, Aggression and Violence

Aggression is defined by social psychologists, as behavior that is intended to harm another individual who does not wish to be harmed [4]. Aggression forms the precursor for violent acts. Aggression can be further divided into physical and nonphysical aggression. Nonphysical aggression includes both verbal and social aggression. Social aggression is when social relationships and status are used to damage reputations and inflict emotional harm on others. Behavior include ostracism, spreading harmful gossip or threats to end an existing relationship [5]. This article focuses on the physical and verbal manifestations of aggression with emphasis on the acute forms of this encountered in the secure inpatient psychiatric wards.

There are two identified trajectories for violent behavior in patients with Schizophrenia and affective psychosis; one which appears directly linked to the positive symptoms and the other where there is associated personality pathology with the illness [6]. One cannot exclude the effects of substance misuse and the likely disrupted social, cognitive skill of mentalizing within this group of patients [7]. Mentalizing is defined as the process by which we can make a sense of each other and ourselves [8]. However, both these can be linked to the above trajectories; whereby the mentalizing function can be impaired in those with personality deficits and the misuse of substances can heighten/escalate the positive symptoms of the condition. We found from our research that most types of hostility or anger on the wards are expressed in the form of verbal aggression [6].

Cornaggia et al [1] conducted a systematic review of aggression with psychiatric wards. They identified 66 studies of unselected psychiatric populations and found the variables most associated with aggression included: impulsiveness/ hostility, previous acts of violence, detention and long duration in hospital (it is not known if the aggression is a consequence of the prolonged admission, related to the severity of the illness or the longer time frame has allowed more time to assess the individual's pattern of aggressive behavior). The aggressor and victim were frequently of the same gender and the younger age of the service user was significant. Less frequent, but prevalent variables were drug/alcohol misuse, diagnosis of a psychotic illness, and the risk of suicide. They further identified twelve studies which explored the aetiology of aggression in those with a psychotic illness. The most relevant factors linked to violence from these studies consisted: substance misuse, hostility, paranoid/persecutory thoughts and acute illness [1].

Papadopoulos et al [9] conducted a meta-analysis to systematically review the types/proportions of antecedents of violence and aggression within the inpatient units. Seventy-one studies were included in this analysis with 59 distinct themes identified, highlighting the wide variety of events which can trigger an aggressive episode. Some of the most common identifiable causal factors from their analysis included; the dynamics between patients and staff with the perceived power imbalance; the restriction of liberties/freedom, frustrations and inadequate communication on the ward can lead to subsequent disdain; patients can also feel that their personal boundaries have been breached on the unit [9].

One in four of incidents concerning violence or aggression have been found to have been triggered by patient-patient interaction. On the locked ward particularly; the impact of having no smoking breaks has been shown to be concordant with increased levels of hostility/aggression on these units [7]. The associated stress with being an inpatient and also visits from loved ones was found to be an antecedent in some cases [9]. The escalation of aggression, following a visit from a family member, has been noticeable in specific patients from our respective wards; particularly when there are high expressed emotions in the dynamics and enmeshed familial relationships.

Prior to a violent incident, patients have been found to display behavioral cues. These were noted to have been the second most frequently reported antecedent of violent incidents [9]. There can be signs of escalating arousal, restlessness or confusion. This would be the time to intervene and pre-empt a serious incident from developing. De-escalation techniques are discussed in the joint British Association for Psychopharmacology (BAP) and National Association of Psychiatric Intensive Care Units (NAPICU) evidencebased consensus guidelines [10].

\section{Interventions that may be Considered in the Locked Ward}

The interactions between patients and staff, along with the culture of the inpatient environment are areas that can be targeted in preventing aggression and violence. Both staff and patients acknowledge and express their dissatisfaction with the provision/ availability of stimulating activities and the design/condition of some inpatient facilities [9]. Patients frequently express their boredom and highlight the paucity of activities/resources accessible in the unit during psychiatric reviews. Interpersonal tension between both groups can disrupt the dynamics and therapeutic milieu of the ward. Poor communication has been cited by patients as a major precursor to an act of aggression/violence [9]. It is visible to others and particularly patient groups when there is discord and impaired harmony/cohesion between staff in the unit. This can be unsettling for the patients and increase their levels of anxiety/tension.

This coupled with the frustrations from poor communication ultimately leads to the discharge and displacement of these through a violent act. The importance of availability, advocating for the service users and building on the therapeutic relationship cannot be downplayed in reducing these acts [9]. The benefits of staff collaboration and good communication can be evidenced from the quality improvement work in some hospitals [11]. Example in East London NHS Foundation trust, there was a forty percent reduction in violence incidents on the ward, following the implementation of simple interventions. A safety huddle, which is utilized in team sports, was introduced. This has been found to promote unity, motivation, planning/strategy, whilst providing clarity regarding introducing roles amongst staff. There was an immediate reduction in violent incidents with this addition. Openness and transparency 
were encouraged in the ward community meetings, where there was a discussion about the violent incidents in the ward, with the aim of moving in a joint valued direction [11].

To target aggression and violence with individual patients one has to consider an idiosyncratic intervention. The biopsychosocial model forms the mainstay of treatment for conditions amongst the spectrum of psychiatric illness and targets individual psychopathology. Psychotropic medication is usually indicated in the management of anger, hostility, aggression and violence. Psychological approaches include the regulation and modulation of emotion in these patients [12]. The importance of early intervention and good risk assessment $[13,14]$ separating the static and dynamic factors (which are targeted in treatment) cannot be emphasized. This along with the incorporation of psychodynamic models forms an inherent aspect in the prevention and reduction of aggression/ hostility in psychiatric settings. The (NAPICU) guidelines specify that secure wards should be homely whilst balancing the safety, security and comforting feel of the unit [15]. They emphasized the benefits of artwork and pictures to circumvent the sometimes chaotic/challenging climate, whilst delineating the therapeutic/ rehabilitative element.

\section{Conclusion}

The aetiology for aggression and violence in secure psychiatric settings is multifactorial. However, small changes in the environment and robust thought out approaches/ good planning by staff can lead to significant reduction in incidence. The impact of violence/aggression is widespread; incidents affect all those involved from the patients/staff to board/senior executives. The financial aftermath is noteworthy both directly (damage to property) and indirectly (Staff Sickness Rates). This is an area which warrants attention and the implementation/testing of new strategies at the individual trust level. Innovation, testing and change remains a key concept of the NHS framework and this is what is required to prevent and manage the concerning episodes of violence/aggression in secure wards.

\section{References}

1. Cornaggia CM, Beghi M, Pavone F, Barale F (2011) Aggression in psychiatry wards: A systematic review. Psychiatry Res 189(1): 10-20.

2. Wehring HJ, Carpenter WT (2011) Violence and schizophrenia. Schizophr Bull 37(5): 877-878.

3. Ahmed SY, Nisha S, Claudius AO, Jude C (2018) Addressing staff morale and wellbeing in psychiatric services. RC Psych in Scotland Blog.

4. Robert AB, Deborah RR (1994) In: (2nd edn), Human aggression. Plenum Press, New York, USA.

5. Brooke LS, Kelly LK, Alexandra Burt (2018) The etiology of social aggression: A nuclear twin family study. Psychol Med 49(1): 162-169.

6. Bo S, Abu Akel A, Kongerslev M, Haahr UH, Simonsen E (2011) Risk factors for violence among patients with schizophrenia. Clinical Psychology Review 31(5): 711-726.

7. Bora E, Yucel M, Pantelis C (2009) Theory of mind impairment in schizophrenia: Meta-analysis. Schizophr Res 109(1-3): 1-9.

8. Anthony B, Peter F (2010) Mentalization based treatment for borderline personality disorder. World Psychiatry 9(1): 11-15.

9. Papadopoulos C, Ross J, Stewart D, Dack C, James K, et al. (2012) The antecedents of violence and aggression within psychiatric in-patient settings. Acta Psychiatr Scand 125(6): 425-439.

10. Patel MX, Sethi FN, Barnes TR, Dix R, Dratcu L (2018) Joint BAP NAPICU evidence-based consensus guidelines for the clinical management of acute disturbance: De-escalation and rapid tranquillization. J Psychopharmacol 32(6): 601-640.

11. Jen TW, Andy C, James I, Brian B, Amar S (2017) Reducing physical violence and developing a safety culture across wards in East London. British Journal of Mental Health Nursing 6(1): 35-43.

12. Zhan J, Ren J, Fan J, Luo J (2015) Distinctive effects of fear and sadness induction on anger and aggressive behavior. Front Psychol 6: 725.

13. National Collaborating Centre for Mental Health (2015) Violence and aggression: Short-term management in mental health, health and community settings. Updated edition. British Psychological Society, London, UK.

14. National Institute for Health and Care Excellence (2017) Violent and aggressive behaviors in people with mental health problems.

15. National Association of Psychiatric Intensive Care and Low Secure Units (NAPICU) (2014) National Minimum Standards for Psychiatric Intensive Care in General Adult Services. 